\title{
Clinicopathological significance of Sox10 expression in triple-negative breast carcinoma
}

\author{
Linfang Jin ${ }^{1,2}$, Chenglin Qin ${ }^{3}$, Xiaowei Qi $^{2}$, Tingting Hong ${ }^{4}$, Xiaodong Yang ${ }^{5}$, Xun Zhu ${ }^{1}$ \\ ${ }^{1}$ Department of Thyroid and Mammary Surgery, The Second affiliated Hospital of Soochow University, Suzhou, China; ${ }^{2}$ Department of Pathology, \\ Affiliated Hospital of Jiangnan University (Wuxi Fourth People's Hospital), Wuxi, China; ${ }^{3}$ Department of General Surgery, The First People's \\ Hospital of Yancheng, Yancheng, China; ${ }^{4}$ Department of Oncology, Affiliated Hospital of Jiangnan University (Wuxi Fourth People's Hospital), \\ Wuxi, China; ${ }^{5}$ Department of Gastrointestinal Surgery, The Second affiliated Hospital of Soochow University, Suzhou, China \\ Contributions: (I) Conception and design: L Jin, X Zhu, X Yang; (II) Administrative support: X Qi ; (III) Provision of study materials or patients: L Jin; \\ (IV) Collection and assembly of data: L Jin, X Zhu, C Qin; (V) Data analysis and interpretation: X Zhu, X Yang; (VI) Manuscript writing: All authors; \\ (VII) Final approval of manuscript: All authors. \\ Correspondence to: Dr. Xun Zhu, MD, PhD. Department of Thyroid and Breast Surgery, The Second affiliated Hospital of Soochow University, 1055 \\ Sanxiang Road, Suzhou 215000, China. Email: 18861530266@163.com; Xiaodong Yang, MD, PhD. Department of Gastrointestinal Surgery, The \\ Second affiliated Hospital of Soochow University, 1055 Sanxiang Road, Suzhou 21500, China. Email: 1040313384@qq.com
}

Background: This study aimed to investigate the SRY-related higll-mobility-group box 10 (Sox10) expression in the pathological diagnosis of triple-negative breast cancer (TNBC). Furthermore, its correlation with the clinicopathological characteristics and disease-free survival rate in patients with TNBC was also evaluated to identify the diagnostic utility of Sox10 as a reliable biomarker for the diagnosis and prognosis of TNBC.

Methods: Using immunohistochemistry (IHC), we identified the expression of Sox10, GATA binding protein 3 (GATA-3), forkhead box protein A1 (FOXA1), gross cystic disease fluid protein (GCDFP15), and mammaglobin (MGB) in 376 cases of primary invasive breast cancer, and 77 cases of metastatic breast cancer. The expression of Sox10 in different molecular subtypes of primary invasive breast cancer and metastatic breast cancer were also compared. Furthermore, the correlation between Sox10 expression and clinicopathological parameters and disease-free survival (DFS) of patients with primary TNBC were also analyzed.

Results: Expression of Sox10 was only detected in the myoepithelial cells of normal breast tissue, but not in any other types of cells, including luminal cells and fibroblasts. The positive rate of Sox10 in primary and metastatic TNBC was significantly higher than that in luminal types and human epidermal growth factor receptor 2 (HER2) overexpressed type. The sensitivity and specificity of Sox10 expression in primary TNBC and metastatic TNBC were significantly lower than GATA-3, significantly higher than FOXA1, GCDFP15, and MGB $(\mathrm{P}<0.001, \mathrm{P}=0.0004, \mathrm{P}=0.0064, \mathrm{P}=0.0229$, respectively). In 71 cases of primary TNBC, a higher expression rate of Sox10 was significantly associated with high-grade tumors, late-stage tumors, and tumors with involvement of four or more lymph node metastases $(\mathrm{P}=0.0145, \mathrm{P}=0.0105, \mathrm{P}=0.0249$, respectively).

Conclusions: Sox10 may be used as a novel reliable putative marker for the diagnosis of TNBC. Notably, Sox10 combined with GATA-3 expression may serve as a supplementary differential diagnostic biomarker for primary and metastatic TNBC. Besides, Sox10 may be a good predictor of the prognosis of primary and metastatic TNBC. This study also highlights the significance of targeting Sox10 as a promising potential therapeutic target gene for TNBC therapy.

Keywords: Triple-negative breast cancer (TNBC); SRY-related higll-mobility-group box 10 (Sox10); clinicopathological characteristics; disease-free survival (DFS)

Submitted Jul 08, 2020. Accepted for publication Aug 28, 2020.

doi: $10.21037 /$ tcr-20-2634

View this article at: http://dx.doi.org/10.21037/tcr-20-2634 


\section{Introduction}

Breast carcinoma is the most prevalent malignancy affecting women worldwide. According to the emerging St. Galen International Expert Consensus on the Primary Therapy of Early Breast Cancer 2017 (1), molecular analysis is difficult to be widely clinically applied due to the high requirement for specimens and high-cost of detection. Therefore, diagnosis based on the findings of immunohistochemistry (IHC) staining remains the gold standard in diagnostics and prognostics of breast carcinoma. The IHC staining method has been extensively applied for the detection of estrogen receptors (ERs) and progesterone receptors (PRs), and Ki67, together with in situ hybridization analysis for human epidermal growth factor receptor 2 (HER2) overexpression or amplification. However, the detection of Ki67 index through IHC staining remains controversial, as it is difficult to define an applicable Ki67 cutoff. The cutoff of Ki67 in our laboratory was defined as $15 \%$. According to ER, PR, HER2, and Ki-67, breast carcinomas are all classified into 4 groups: luminal A (ER and/or PR positive, and PR $\geq 20 \%$, HER2 negative, Ki67 $<15 \%$ ), luminal B (ER and/or PR positive, HER2 negative and Ki67 $\geq 15 \%$, or PR <20\%; ER and/or PR positive, HER2 positive), HER2 overexpressed (HER2 positive) and triple-negative (ER negative, PR negative and HER2 negative). Triple-negative breast cancer (TNBC), a specific molecular subtype of breast cancer, is predominantly characterized by the lack of expression of targeted biomarkers including ER, PR, and HER2. Accumulating evidence has shown that patients with TNBC account for $10-15 \%$ of all breast carcinoma cases; however, Hispanic and African-American women paradoxically represent the majority of cases (2). Notably, in China, nearly $12-20 \%$ of all the breast carcinoma diagnoses are TNBC, which is considerably higher compared to Western countries (3). Characteristically, TNBC is highly aggressive and involves poorly differentiated tumors that exhibit higher recurrence and metastatic rates and a worse overall prognosis than the other subtypes of breast carcinoma. Thus, TNBC has become the major focus of breast carcinoma research (2). More recently, it has been established that there is considerable heterogeneity in natural history and prognosis within TNBC cases, which results in the exhibiting of different clinicopathological characteristics. Because TNBC is prone to distant metastasis and does not express ER and PR, it presents a formidable challenge to the pathologist in differentiating it from metastatic adenocarcinomas originating from other sites.
Moreover, in clinical practice, it can be difficult to establish the origin of a putative breast metastasis if the primary tumor is either triple-negative for breast biomarkers (ER/ PR/HER2), or if there is a loss of biomarker expression in the metastasis. Therefore, novel molecular targets with high sensitivity and specificity for TNBC are highly desirable to facilitate timely and accurate diagnosis of primary or metastatic TNBC, and assist in appropriately classifying breast carcinomas.

The ancillary technique of IHC has become an indispensable tool in anatomic pathology. Tissuespecific IHC markers used in conjunction with histologic morphology are particularly beneficial in identifying tumors of unknown origin. To date, numerous immunohistochemical markers have been suggested to be beneficial in assisting the histopathological diagnosis of breast carcinoma, and many biomarkers have gained acceptance and translated into routine use. Among them, gross cystic disease fluid protein 15 (GCDFP-15) and mammaglobin (MGB) serve as the most commonly applied breast-specific immunohistochemical markers in the diagnosis of breast carcinoma or metastatic breast carcinoma (4). The marker GCDFP-15 has been recognized as a major constituent protein in breast cyst fluid and may be expressed in cells with characteristics similar to sweat glands, including acinar and salivary glands, skin, and vulva. Besides that in breast carcinoma, GCDFP-15 expression has been well documented in only a few cancers, including prostate, and skin adnexal cancers (5). Predominantly, GCDFP-15 exhibits high sensitivity and specificity for breast carcinoma and is often used as an effective immunohistochemical marker to determine the potential source of breast metastases in carcinomas with unknown primary sites.

The marker MGB is a member of the secretoglobin family of proteins, with a predicted molecular weight of $10 \mathrm{kD}$ as cloned from breast cancer epithelial cells (6). The protein has been suggested as a tumor-associated marker for the diagnosis of breast carcinoma (7). Positive expression of GCDFP-15 and MGB in breast carcinomas is $50 \%$ and $75 \%$, respectively; but their sensitivity remains poor, particularly in high-grade breast cancer such as triplenegative breast carcinoma (4-7).

The marker GATA-3 belongs to the GATA family of zinc finger protein transcription factors. It plays crucial role in mammary development and luminal epithelial cell differentiation (8), as well as mediating the development, proliferation, and differentiation of a wide variety of other 
tissue types including kidney (9), nervous system (10), T cells (11), and hair follicles (12). In the luminal A subtype of breast cancer, GATA-3 is highly expressed (13). There is increasing evidence that GATA-3 is a potential tool for differentiating primary and metastatic breast carcinomas (14-18).

Forkhead box protein A1 (FOXA1) is a member of the forkhead box gene family of winged-helix transcription factors. It plays a pivotal role in the regulation of organogenesis of many organs, and is closely associated with the pathogenesis of a number of cancer types, including breast carcinoma (19). The expression of FOXA1 is associated with molecular subtypes and may be indicative of a good prognosis in breast carcinoma (19). Moreover, it also regulates the expression level of ERa in breast carcinoma (20).

The protein SRY-related high-mobility-group box 10 (Sox10) is a member of the Sox transcription factor family and mediates the differentiation of neural crest cells into melanocytes, oligodendrocytes, and glia, and promotes a mesenchymal transition in the mammary cell. It is expressed in many different cells and tissues including Schwann cells of peripheral nerves, epidermal melanocytes, oligodendrocytes of the cerebral cortex, mast cells, myoepithelial cells of submucosal bronchial glands, and acinar of mammary and corresponding tumors. Immunohistochemical expression of Sox10 is also present in melanoma, peripheral nerve sheath tumors, and salivary gland myoepithelioma (21). Recently, it has been reported that Sox10 is highly expressed in primary breast carcinomas, predominantly by basal-like breast carcinomas and unclassified TNBC $(22,23)$ in limited literature.

Based on the findings of the literature review, we hypothesized that since the expression rate of Sox 10 in TNBC is considerably higher than in other types of breast carcinoma, it could possibly be used as a putative biomarker for TNBC. Therefore, in this study aimed to evaluate the expression of Sox10 in TNBC, and compare the specificity and sensitivity of Sox10 with GATA-3, FOXA1, GCDFP15, and MGB in TNBC, in order to screen out the most helpful markers for TNBC diagnosis. In addition, there are few studies on the relationship between Sox10 and the clinicopathological characteristics, 5-year DFS of TNBC. Furthermore, the correlation between Sox10 protein expression and clinicopathological characteristics of primary TNBC was also determined to evaluate its prognostic significance in TNBC.

We present the following article in accordance with the
REMARK reporting checklist (available at http://dx.doi. org/10.21037/tcr-20-2634).

\section{Methods}

\section{Specimens}

Our study included tumor specimens from primary invasive breast carcinomas (only ductal and lobular cancers; $n=376$ ), metastatic breast carcinomas (only ductal and lobular cancers; $\mathrm{n}=77,30$ in lymph nodes, 15 in liver, 16 in lung and pleura, 5 in brain, 10 in bone, 1 in adrenal), and 20 normal breast tissue specimens were also collected. Tumor tissues of primary invasive breast carcinomas were collected from biopsies and surgical resections from the Department of Pathology, Affiliated Hospital of Jiangnan University, between January 2010 and June 2013. Tumor tissues from metastatic breast carcinomas and normal breast tissues were obtained between January 2016 and September 2018. None of the patients with primary invasive breast carcinomas had received preoperative chemotherapy or radiotherapy. The patients with metastatic breast carcinomas received surgical therapies with their primary focus. Hematoxylin and eosin (H\&E) stained tissue sections and IHC staining of all specimens were reviewed by two experienced and qualified pathologists in our hospital. All the cases included had complete clinicopathological data. The collected tissue from primary invasive breast cancer groups and metastatic breast carcinomas were all classified into 3 groups: luminal (A and B) $(\mathrm{n}=240)$, HER2 overexpressed $(\mathrm{n}=65)$, and triple-negative $(\mathrm{n}=71)$; luminal (A and B) $(\mathrm{n}=44)$, HER2 overexpressed $(\mathrm{n}=11)$, triple-negative $(\mathrm{n}=22)$, respectively. There were 71 cases of primary TNBC which were followed-up from the beginning of surgery. As of October 2018, the followup period was at 5 years. There were 25 cases of primary TNBC which exhibited disease-free survival, and 46 cases had recurred or metastasized (including 12 cases of simple liver metastasis, 13 cases of simple lung metastasis, 6 cases of simple bone metastasis, 7 cases of simple brain metastasis, 2 cases of soft tissue metastasis, and 6 cases of multiple metastases). Of these, 23 patient cases died due to metastasis.

The clinical staging criteria for primary breast carcinoma were based on the clinical staging criteria recommended by the Union for International Cancer Control (UICC) and the United States Joint Commission for Cancer (AJCC). Tumor histological classification and differentiation grades were determined based on the Nottingham Combined Histology Grade. 
Table 1 Primary antibody information

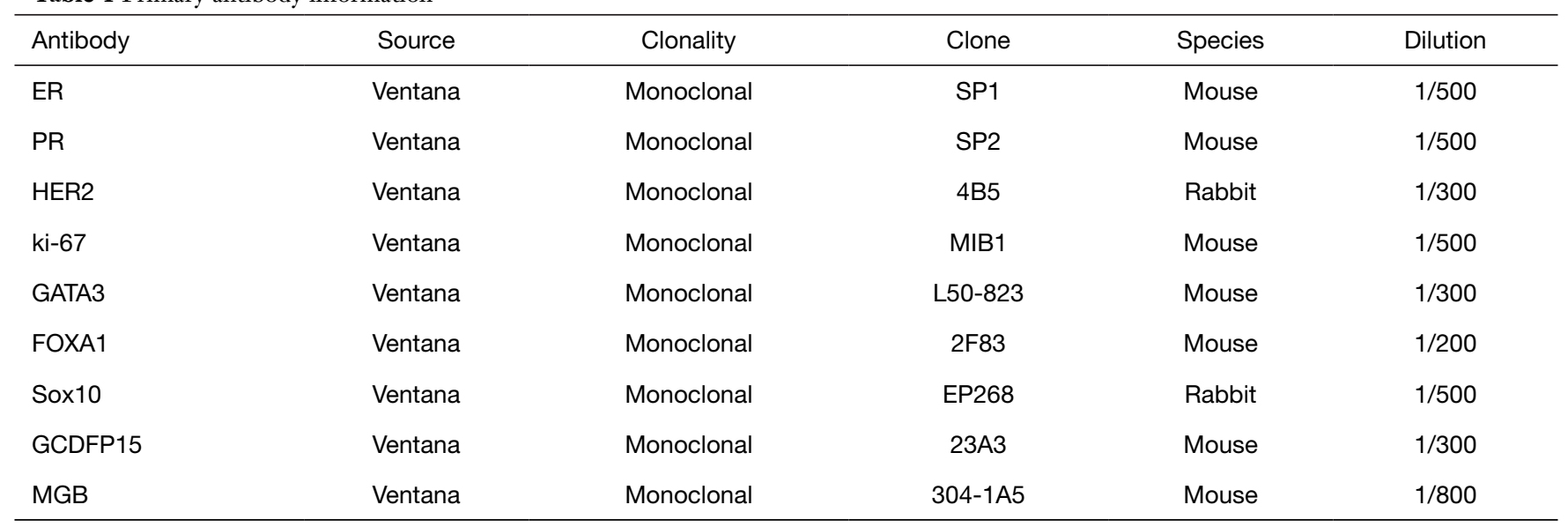

This work was approved by the Hospital Ethical Review Committee of Jiangnan University (No. LS2016022). It was decided by the committees that written informed consent was not required for the present study from each patient. The study was conducted in accordance with the Declaration of Helsinki (as revised in 2013).

\section{IHC}

For IHC analysis, tissue specimens were fixed in $10 \%$ formalin, embedded in paraffin, dehydrated in an ethanol gradient, and then were serially sectioned into a $4-\mu \mathrm{m}$ thickness. H\&E staining was performed by experienced pathologists to verify the pathological diagnosis. Immunostaining was performed on the sections following a standard immunostaining protocol using a Ventana BenchMark XT automated stainer (Roche, Basel, Switzerland). Briefly, sections were dewaxed in xylene and hydrated in an ethanol gradient. Antigen retrieval was performed by heating the tissue sections at $100{ }^{\circ} \mathrm{C}$ for $30 \mathrm{~min}$ in a citrate buffer. Moreover, endogenous peroxidase activity was blocked by incubation in 3\% hydrogen peroxide for $10 \mathrm{~min}$. Subsequently, the sections were incubated in $5 \%$ bovine serum albumin (BSA) at room temperature for $30 \mathrm{~min}$. Primary antibodies ER, PR, ki-67, GATA-3, FOXA1, Sox10, GCDFP15, MGB monoclonal antibodies (Gene Tech, Shanghai, China), and HER2 monoclonal antibody (Roche, Basel, Switzerland), were respectively added drop-wise, followed by overnight incubation at $4{ }^{\circ} \mathrm{C}$. The slides were washed and incubated with a horseradish peroxidase-conjugated secondary antibody for $30 \mathrm{~min}$. The immunostaining was carried out by staining with
3, 3'-diaminobenzidine tetrahydrochloride solution (GTVisionII Immunohistochemistry Detection Kit for Rabbit/Mouse, Gene Tech, Shanghai, China), counterstaining with hematoxylin, dehydrated and mounted, and the sections were then examined under a microscope. Pertinent information concerning the primary antibodies is summarized in Table 1. Simultaneously, sections recognized to stain positively were used as a positive control. For the negative control, the primary antibody was replaced with phosphate-buffered saline. Fluorescence in situ hybridization (FISH) was evaluated using the PathVysion HER2 DNA Probe Kit (Abbott-Vysis, Chicago, USA).

\section{Decision criteria}

The markers ER, PR, ki67, GATA-3, FOXA1, Sox10 were localized in the nucleus, HER2 in the membrane, and GCDFP15 and MGB in the cytoplasm. The positive staining signal was brown and yellow. The H\&E stained and immunostained sections were reviewed independently by two pathologists to obtain a consensual score. In each section, five visual fields were selected. The immunostaining for ER, PR, GATA-3, FOXA1, Sox10, GCDFP15, and MGB was considered positive when more than $1 \%$ of tumor cells were stained positive. The guidelines for IHC testing of HER2 were adopted according to the American Society of Clinical Oncology (ASCO) and the College of American Pathologists (CAP) in 2018.

\section{Statistical analysis}

Statistical analyses were performed using the SPSS 23.0 

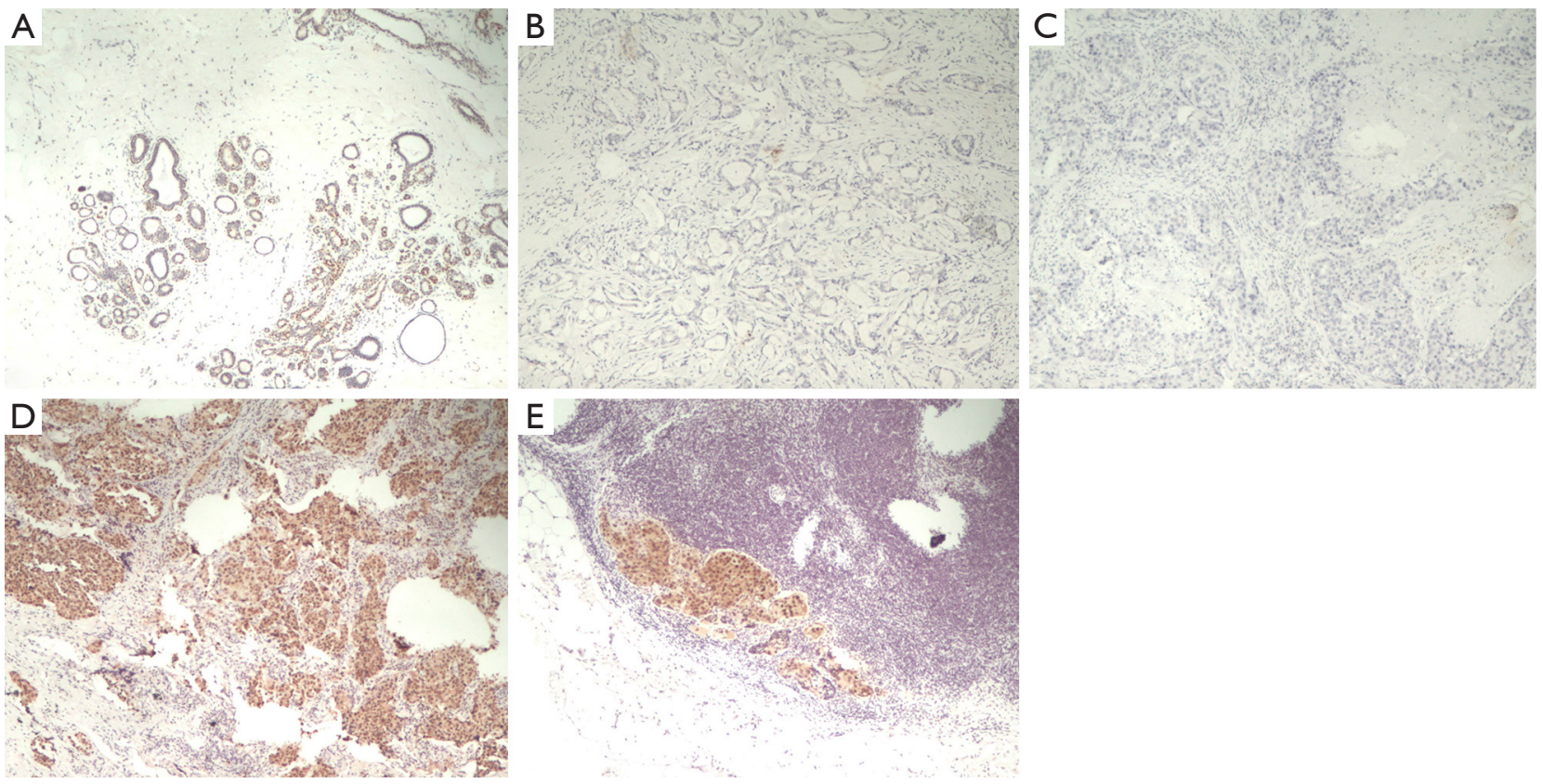

Figure 1 The luminal cells and stromal fibroblasts showing negative immunostaining for Sox10, as well as the myoepithelium showing positive immunostaining in normal breast tissues (A, 40x); Sox10-negative luminal and HER2 overexpressed breast carcinoma (B,C, 40x); Sox10-positive primary and metastatic TNBC (D,E, 40x). Sox10, SRY-related higll-mobility-group box 10; HER2, human epidermal growth factor receptor 2; TNBC, triple-negative breast cancer.

software (SPSS Inc., Chicago, IL, USA). Fisher 2-tailed exact tests were performed to compare the differences between groups. Pearson's correlation was used to analyze the association between IHC markers and the clinicopathological data. Disease-free survival (DFS) was plotted using the Kaplan-Meier method and was compared using the log-rank test. Statistical significance was set at $\mathrm{P}<0.05$.

\section{Results}

\section{Expression of Sox10 in normal breast tissue}

The positive rate of Sox10 in the myoepithelium of 20 normal breast tissues was $100 \%$ (20/20). Expression of Sox10 was not observed in breast luminal cells and stromal fibroblasts (Figure 1).

\section{Comparison of Sox10 expression among different molecular subtypes of primary breast cancer and metastatic breast carcinoma}

The positive rates of Sox10 in luminal type, HER2 overexpressing type, and TNBC were $0 \%, 3.1 \%$, and $67.6 \%$, respectively. The positive rates of Sox 10 in metastatic breast cancer in luminal type, HER2 overexpressing type, and TNBC were $0 \%, 9.1 \%$, and $68.2 \%$, respectively. The positive rate of Sox 10 expression in primary and metastatic TNBC was significantly higher than that in other molecular subtypes $(\mathrm{P}<0.001, \mathrm{P}<0.001)$ (Table 2).

\section{Sensitivity and specificity of Sox10 and GATA-3, FOXA1, GCDFP15, and MGB in primary TNBC and metastatic $T N B C$}

The sensitivity and specificity of Sox 10 and GATA-3 in primary TNBC were $67.6 \%$ and $31.6 \%, 87.3 \%$ and $40.8 \%$, respectively. The sensitivity and specificity of Sox10 and GATA-3 in metastatic TNBC were $68.2 \%$ and $32.6 \%$, $86.4 \%$ and $41.3 \%$, respectively. Compared with other markers, the sensitivity and specificity of Sox 10 in primary and metastatic TNBC were only significantly lower than GATA-3, and were significantly higher than FOXA1, GCDFP15, and MGB $(\mathrm{P}<0.001, \mathrm{P}=0.0004, \mathrm{P}=0.0064$, and $\mathrm{P}=0.0229$, respectively) (Table 3). 
Table 2 Comparison of Sox10 expression among different molecular subtypes of primary breast cancer and metastatic breast carcinoma

\begin{tabular}{|c|c|c|c|c|c|c|}
\hline & \multicolumn{3}{|c|}{ Primary breast cancer } & \multicolumn{3}{|c|}{ Metastatic breast carcinoma } \\
\hline Luminal $(A, B)$ & $0(0 \%)$ & $240(100 \%)$ & 240 & $0(0 \%)$ & $44(100 \%)$ & 44 \\
\hline HER2 & $2(3.1 \%)$ & $63(96.9 \%)$ & 65 & $1(9.1 \%)$ & $10(90.9 \%)$ & 11 \\
\hline TNBC & $48(67.6 \%)$ & $23(32.4 \%)$ & 71 & $15(68.2 \%)$ & $7(31.8 \%)$ & 22 \\
\hline$\chi^{2}$ & \multicolumn{3}{|c|}{359.047} & \multicolumn{2}{|c|}{71.263} & \\
\hline$P$ value & \multicolumn{2}{|c|}{$<0.001$} & & \multicolumn{2}{|c|}{$<0.001$} & \\
\hline
\end{tabular}

Table 3 Sensitivity and specificity of Sox10 and GATA-3, FOXA1, GCDFP15, and MGB in primary TNBC and metastatic TNBC

\begin{tabular}{|c|c|c|c|c|c|c|c|c|}
\hline & \multicolumn{4}{|c|}{ Primary TNBC } & \multicolumn{4}{|c|}{ Metastatic TNBC } \\
\hline Sox10 & $48(67.6 \%)$ & $23(32.4 \%)$ & $67.6 \%(48 / 71)$ & $31.6 \%(48 / 152)$ & $15(68.2 \%)$ & 7 (31.8\%) & $68.2 \%(15 / 22)$ & $32.6 \%(15 / 46)$ \\
\hline FOXA1 & $21(29.6 \%)$ & $50(70.4 \%)$ & $29.6 \%(21 / 71)$ & $13.8 \%(21 / 152)$ & $4(18.2 \%)$ & $18(81.8 \%)$ & $18.2 \%(4 / 22)$ & $8.7 \%(4 / 46)$ \\
\hline GATA-3 & $62(87.3 \%)$ & 9 (12.7\%) & $87.3 \%(62 / 71)$ & $40.8 \%(62 / 152)$ & $19(86.4 \%)$ & $3(13.6 \%)$ & $86.4 \%(19 / 22)$ & $41.3 \%(19 / 46)$ \\
\hline MGB & $14(19.7 \%)$ & 57 (80.3\%) & $19.7 \%(14 / 71)$ & $9.2 \%(14 / 152)$ & 5 (22.7\%) & $17(77.3 \%)$ & $22.7 \%(5 / 22)$ & $10.9 \%(5 / 46)$ \\
\hline $\mathrm{n}$ & 152 & 203 & & & 46 & 64 & & \\
\hline$\chi^{2}$ & & & 19.06 & 12.67 & & & 7.42 & 5.17 \\
\hline$P$ value & & & $<0.001$ & 0.0004 & & & 0.0064 & 0.0229 \\
\hline
\end{tabular}

Correlation between the expression of Sox 10 and clinicopathological characteristics of primary triple-negative breast carcinoma

For primary TNBC, the expression of Sox 10 was significantly correlated with the pathological grade of the tumors. The positive rate of Sox10 in high-grade tumors was significantly higher than that in middle-grade tumors $(\mathrm{P}=0.0160)$. The expression of Sox10 was also significantly associated with clinical stages. The positive rate of Sox10 in late-stage tumors was higher than that in early- and middlestage tumors $(\mathrm{P}=0.0105)$. Moreover, Sox10 expression was also significantly correlated to lymph node metastasis. The positive rate of Sox10 in tumors with four or more lymph node metastases was higher than that in tumors without lymph node metastasis and with 1-3 lymph node metastases $(\mathrm{P}=0.0249)$. However, there was no significant correlation with other clinicopathological characteristics of primary TNBC including the age of onset, tumor diameter, and pathological type (Table 4).

Correlation between the expression of Sox10 and the prognosis of primary triple-negative breast carcinoma

Kaplan-Meier survival analysis showed that the expression of Sox10 was associated with DFS in patients with primary TNBC. In 71 cases of primary TNBC, the DFS time of the Sox10 positive group was significantly lower than that of the negative group $(\mathrm{P}=0.00008)$ (Figure 2).

\section{Discussion}

With the substantial increase in the incidence of breast carcinoma, ever increasing numbers of women are suffering from breast cancer. Breast carcinoma is a highly heterogeneous disease and constitutes different molecular subtypes, which are HER2, luminal A, luminal B, claudinlow, and basal-like types. The majority TNBCs are included 
Table 4 Correlation between the expression of Sox10 and clinicopathological characteristics of primary triple-negative breast carcinoma.

\begin{tabular}{|c|c|c|c|c|c|}
\hline \multirow{2}{*}{ Clinico-pathological parameters } & \multirow{2}{*}{$\mathrm{n}=71$} & \multicolumn{2}{|c|}{ Sox10 } & \multirow{2}{*}{$\chi^{2}$} & \multirow{2}{*}{$P$ value } \\
\hline & & + & - & & \\
\hline Age & & & & 0.42 & 0.5150 \\
\hline$<59$ & 44 & 28 & 16 & & \\
\hline$\geq 59$ & 27 & 20 & 7 & & \\
\hline III & 45 & 35 & 10 & & \\
\hline Stage & & & & 6.54 & 0.0105 \\
\hline $\mid-I I$ & 28 & 14 & 14 & & \\
\hline$>2$ & 43 & 32 & 11 & & \\
\hline Lymph node metastasis & & & & 7.39 & 0.0249 \\
\hline 0 & 46 & 29 & 17 & & \\
\hline $1-3$ & 16 & 14 & 2 & & \\
\hline$\geq 4$ & 9 & 9 & 0 & & \\
\hline Morphology & & & & 1.71 & 0.1915 \\
\hline Ductal carcinoma & 69 & 48 & 21 & & \\
\hline Lobular carcinoma & 2 & 0 & 2 & & \\
\hline
\end{tabular}

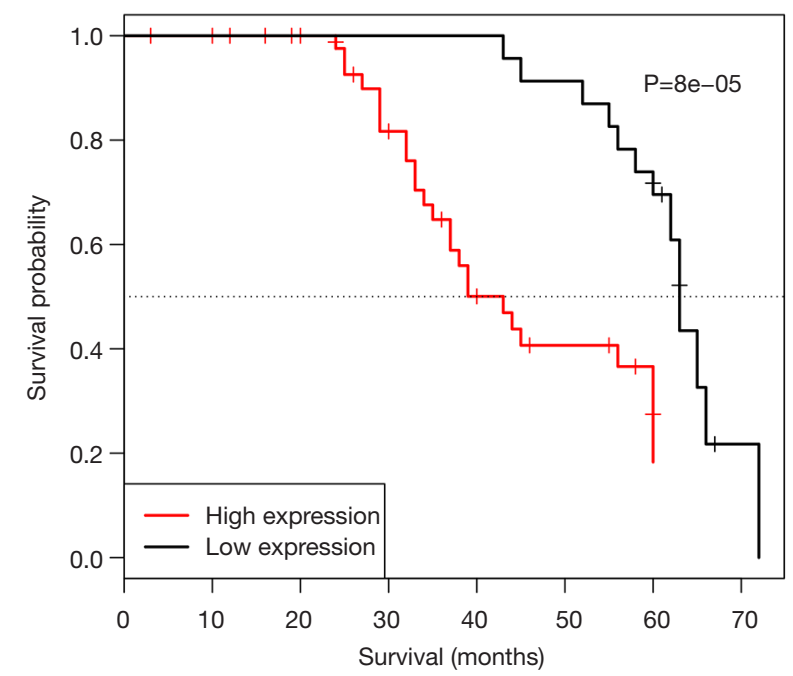

Figure 2 Correlation between the expression of Sox10 and the DFS of TNBC. P=0.00008. Sox10, SRY-related higll-mobilitygroup box 10; DFS, disease-free survival; TNBC, triple-negative breast cancer. in the basal-like subtype. The different molecular types of breast cancer exhibit distinct molecular mechanisms and act as biologically distinct entities that require different therapeutic management. Survival analysis has revealed a poor prognosis in patients diagnosed with basal-like type, while the two ER+ groups have shown a variable outcome.

When we encounter TNBC, particularly metastatic TNBC, it is often difficult to diagnose. Therefore, apart from recognition of the clinical presentation, some specific immunohistochemical markers are needed to further confirm the diagnosis. Presently, ER, GCDFP15, and MGB are the most commonly used markers in the diagnosis of breast carcinoma. However, recently an increasing number of studies have considered GATA-3 as a useful marker in breast carcinoma (13-18). The transcription factor FOXA1 is known for molecular subtyping of breast carcinoma, its expression is associated with good clinical outcomes and is also considered as a molecular marker for breast carcinoma diagnostics (19). More recently, Sox10 with more than 20 
SOX family transcription factors has been characterized by a high mobility group (HMG) DNA binding domain sequence and recognized to play a crucial role in the development of the nervous system, immune system, and skeletal system (21).

Existing studies have suggested that Sox10 plays an important role in assisting the diagnosis of melanoma, peripheral nerve-derived tumor, and salivary gland myoepithelial tumors (22). Notably, Sox10 is a sensitive lineage marker for both primary and metastatic TNBC; however, the diagnostic value of Sox 10 in TNBC remains to be elucidated. The Notch signaling pathway plays a central role in maintaining stem cell characteristics and regulating cell differentiation in breast tissue (24). The Notch gene is usually activated in glandular precursor cells of breast lobules (25). In vitro studies on mouse mammary epithelial cells revealed that Notch4-PBP mediated cell proliferation required the involvement of Sox10, however, the other roles of Sox10 in the differentiation and development of mammary epithelial cells remain elusive (26). Studies showed that Sox10 was only expressed in myoepithelial cells of normal breast tissue by IHC method, but not in other cells (27). In this study, we also found that Sox10 was only expressed in the myoepithelial cells around the lobules and ducts in normal breast tissue, but not in the other lumen cells. Consistently, it was also localized in the nucleus, as reported previously. Myoepithelial cells exist not only in the breast but also in many other tissues, including soft tissue and salivary gland. The expression of Sox10 was detected in salivary gland myoepithelial tumors such as acinar cell carcinoma, adenoid cystic carcinoma, myoepithelial carcinoma, and epithelial-myoepithelial carcinoma; however, not in mucoepidermoid carcinoma and ductal carcinoma from non-myoepithelial origin $(28,29)$. Miettinen et al. (30) also suggested that Sox10 was not only a diagnostic marker for schwannoma and malignant melanoma, but also expressed in myoepithelial tumors in soft tissue. Considering the results from these studies, we can conclude that Sox10 is a good diagnostic marker for myoepithelial tumors, and the expression of Sox10 might suggest that the tumors may originate from myoepithelium.

Furthermore, Cimino-Mathews (23), Ivanov (28), and others have found that Sox10 can be expressed in primary breast carcinoma, predominantly in basal-like breast carcinoma and unclassified TNBC. Recently, an IHC based study (31) has demonstrated Sox10 to be an additional marker in TNBC, especially for the basallike and metaplastic subtypes. Similarly, we also found a significantly higher positive rate of Sox10 in primary and metastatic TNBC than in the other two types $(\mathrm{P}<0.001$, $\mathrm{P}<0.001$, respectively), which was consistent with the findings of the previously reported literature. The TNBC is considered to be a malignant tumor originating from breast myoepithelial cells, which explains why Sox10 is more positively expressed in TNBC than in other types of breast carcinoma. The characteristic of TNBC is that ER, PR, and HER2 are not expressed and the heterogeneity is very high. The three markers characteristically exhibit a negative profile in TNBC, therefore, treatment of this molecular sub-type remains highly challenging. Consequently, a large number of studies on TNBC have mainly focused on deciphering novel therapeutic targets and strategies. Pathological diagnosis of TNBC remains the gold standard for pathological diagnosis. Since ER, PR, and HER2 are not expressed in TNBC, these three markers cannot be used to differentiate it from metastatic adenocarcinoma. In addition, through literature reviews and from this study, we demonstrated that besides GATA-3, FOXA1, GCDFP15, and MGB, which are the most extensively used markers for routine diagnosis of breast carcinoma, exhibit low sensitivity and specificity in distinguishing primary TNBC and metastatic TNBC. Thus, by comparing the sensitivity and specificity of expression of Sox 10 with that of GATA-3, FOXA1, GCDFP15, and MGB in TNBC, this study revealed that the sensitivity and specificity of Sox10 were significantly higher than those of the other three markers $(\mathrm{P}<0.001, \mathrm{P}=0.0004, \mathrm{P}=0.0064, \mathrm{P}=0.0229$, respectively) for both primary and metastatic TNBC. Therefore, this indicated that the sensitivity and specificity of Sox 10 for TNBC were remarkably high. In routine immunohistopathology, expression of Sox 10 combined with GATA-3 may serve as a promising putative IHC marker for the diagnosis of TNBC, particularly for metastatic TNBC.

Accumulating studies have suggested that Soxl0 plays an important role in the occurrence and development of various tumors, and participates in the proliferation, migration, and invasion of tumor cells (32-34). Owing to differences in the expression level of Sox 10 in a variety of tumors, its effect on cell function is also differently attributable to differences in mechanisms. Overexpression of Sox10 happens in nasopharyngeal carcinoma (32) and bladder cancer (33), and this promotes the growth and metastasis of tumors and plays an oncogenic role. However, in many digestive tract tumors, the expression of Sox10 is low and it plays the role of tumor suppressor (34). However, presently there are limited clinical studies on the role of 
Sox10 in the occurrence and development of primary TNBC. By analyzing the correlation between the expression of Sox10 protein and clinicopathological characteristics in 71 cases of primary TNBC, our study revealed that the positive rate of Sox 10 was significantly correlated with pathological grade, clinical stage, and a number of lymph node metastases. The positive rate of Sox10 expression was higher in high-grade TNBC compared to low-grade TNBC $(\mathrm{P}=0.0145)$, the positive rate of Sox10 expression in late-stage TNBC was remarkably higher than that of early-stage TNBC $(\mathrm{P}=0.0105)$, and the positive rate of Sox10 expression was significantly higher in cases with lesser numbers of lymph node metastases than those with greater numbers of lymph node metastases $(\mathrm{P}=0.0249)$. Based on these findings, we speculated that Sox10 also plays an oncogenic role in the occurrence and development of TNBC. The prognosis of tumors is related to many factors, including the stage of the tumors, the number of lymph node metastases, pathological grading, and tumor size. The later the stage, the worse the prognosis of the tumors; the higher the pathological grade, the worse the prognosis; similarly, a higher number of lymph node metastases is also considerably associated with the prognosis of TNBC. There is a growing body of evidence which demonstrates that the more involvement of the lymph node, the more prone the tumors are to metastasis and recurrence. Since the expression of the Sox10 protein was correlated with tumor stage, number of lymph node metastases, and pathological grade of primary TNBC in this study, the study indicated that the expression of Sox10 protein is highly associated with the adverse prognosis of primary TNBC. Thus, Sox 10 may serve as a novel putative biomarker for predicting the prognosis and metastasis of primary TNBC and potential target genes for the treatment of TNBC. Further followup of the patients who had primary TNBC revealed that the 5-year DFS period of those with positive expression of Sox10 protein was significantly lower than that with the negative expression $(\mathrm{P}=0.00008)$. This finding further confirmed our hypothesis that the high expression of Sox 10 can be used as an index to predict the prognosis of patients with TNBC. Conceivably, the high expression of Sox10 was an independent predictor of TNBC. Thus, identifying the expression of Sox10 through the IHC technique can be used as a supplementary diagnostic marker for risk prediction.

In this study, the sample size may be a little small. In the next step, we will increase the sample size and carry out further studies at the level of in vitro cytology to increase the possible mechanism analysis. And maybe this will be more conducive to support the conclusions of this study.

\section{Conclusions}

The findings of this preliminary study suggested that Sox10 expression is a novel prognostic biomarker for TNBC, and highlighted the clinical significance of targeting Sox10 as a promising potential therapeutic target gene for TNBC therapy. Further studies recruiting a larger sample size are warranted at RNA levels to more firmly establish these findings.

\section{Acknowledgments}

The authors acknowledge Mr. Longhai Li and Lihua Zhang for statistical analysis.

Funding: This work was supported in part by a grant from Wuxi Scientific and Technology Bureau ("Wuxi Scientific and Technology Development Funding”, CSE31N1710).

\section{Footnote}

Reporting Checklist: The authors have completed the REMARK reporting checklist. Available at http://dx.doi. org/10.21037/tcr-20-2634

Data Sharing Statement: Available at http://dx.doi. org/10.21037/tcr-20-2634

Conflicts of Interest: All authors have completed the ICMJE uniform disclosure form (available at http://dx.doi. org/10.21037/tcr-20-2634). The authors have no conflicts of interest to declare.

Ethical Statement: The authors are accountable for all aspects of the work in ensuring that questions related to the accuracy or integrity of any part of the work are appropriately investigated and resolved. This work was approved by the Hospital Ethical Review Committee of Jiangnan University (No. LS2016022). It was decided by the committees that written informed consent was not required for the present study from each patient. The study was conducted in accordance with the Declaration of Helsinki (as revised in 2013).

Open Access Statement: This is an Open Access article distributed in accordance with the Creative Commons Attribution-NonCommercial-NoDerivs 4.0 International 
License (CC BY-NC-ND 4.0), which permits the noncommercial replication and distribution of the article with the strict proviso that no changes or edits are made and the original work is properly cited (including links to both the formal publication through the relevant DOI and the license). See: https://creativecommons.org/licenses/by-nc-nd/4.0/.

\section{References}

1. Curigliano G, Burstein HJ, Winer EP, et al. De-escalating and escalating treatments for early-stage breast cancer: the St. Gallen International Expert Consensus Conference on the Primary Therapy of Early Breast Cancer 2017. Ann Oncol 2017;28:1700-12.

2. Bidard FC, Conforti R, Boulet T, et al. Does triplenegative phenotype accurately identify basal-like tumour? An immunohistochemical analysis based on 143 'triplenegative' breast cancers. Ann Oncol 2007;18:1285-6.

3. Wang RX, Xu XE, Huang L, et al. eEF2 kinase mediated autophagy as a potential therapeutic target for paclitaxelresistant triple-negative breast cancer. Ann Transl Med 2019;7:783.

4. Darb-Esfahani S, von Minckwitz G, Denkert C, et al. Gross cystic disease fluid protein 15 (GCDFP-15) expression in breast cancer subtypes. BMC Cancer 2014;14:546.

5. Lewis GH, Subhawong AP, Nassar H, et al. Relationship between molecular subtype of invasive breast carcinoma and expression of gross cystic disease fluid protein 15 and mammaglobin. Am J Clin Pathol 2011;135:587-91.

6. Zehentner BK, Carter D. Mammaglobin: a candidate diagnostic marker for breast cancer. Clin Biochem 2004;37:249-57.

7. Bozhenko VK, Kharchenko NV, Vaskevich EF, et al. Mammaglobin in peripheral blood and tumor in breast cancer patients. Biomed Khim 2016;62:453-7.

8. Kouros-Mehr H, Kim JW, Bechis SK, et al. GATA-3 and the regulation of the mammary luminal cell fate. Curr Opin Cell Biol 2008;20:164-70.

9. Grote D, Souabni A, Busslinger M, et al. Pax2/8-regulated Gata3 expression is necessary for morphogenesis and guidance of the nephric duct in the developing kidney. Development 2006;133:53-61.

10. Lim KC, Lakshmanan G, Crawford SE, et al. Gata3 loss leads to embryonic lethality due to noradrenaline deficiency of the sympathetic nervous system. Nat Genet 2000;25:209-12.

11. Ting CN, Olson MC, Barton KP, et al. Transcription factor GATA-3 is required for development of the T-cell lineage. Nature 1996;384:474-8.

12. Kaufman CK, Zhou P, Pasolli HA, et al. GATA-3: an unexpected regulator of cell lineage determination in skin. Genes Dev 2003;17:2108-22.

13. Usary J, Llaca V, Karaca G, et al. Mutation of GATA3 in human breast tumors. Oncogene 2004;23:7669-78.

14. Miettinen M, McCue PA, Sarlomo-Rikala M, et al. GATA3: a multispecific but potentially useful marker in surgical pathology: a systematic analysis of 2500 epithelial and nonepithelial tumors. Am J Surg Pathol 2014;38:13-22.

15. Liu H, Shi J, Wilkerson ML, et al. Immunohistochemical evaluation of GATA3 expression in tumors and normal tissues: a useful immunomarker for breast and urothelial carcinomas. Am J Clin Pathol 2012;138:57-64.

16. Krings G, Nystrom M, Mehdi I, et al. Diagnostic utility and sensitivities of GATA3 antibodies in triple-negative breast cancer. Hum Pathol 2014;45:2225-32.

17. Braxton DR, Cohen C, Siddiqui MT. Utility of GATA3 immunohistochemistry for diagnosis of metastatic breast carcinoma in cytology specimens. Diagn Cytopathol 2015;43:271-7.

18. Wendroth SM, Mentrikoski MJ, Wick MR. GATA3 expression in morphologic subtypes of breast carcinoma: a comparison with gross cystic disease fluid protein 15 and mammaglobin. Ann Diagn Pathol 2015;19:6-9.

19. Badve S, Turbin D, Thorat MA, et al. FOXA1 expression in breast cancer--correlation with luminal subtype A and survival. Clin Cancer Res 2007;13:4415-21.

20. Carroll JS, Liu XS, Brodsky AS, et al. Chromosome-wide mapping of estrogen receptor binding reveals long-range regulation requiring the forkhead protein FoxA1. Cell 2005;122:33-43.

21. Ordóñez NG. Value of SOX10 immunostaining in tumor diagnosis. Adv Anat Pathol 2013;20:275-83.

22. Nonaka D, Chiriboga L, Rubin BP. Sox10: a panschwannian and melanocytic marker. Am J Surg Pathol 2008;32:1291-8.

23. Cimino-Mathews A, Subhawong AP, Elwood H, et al. Neural crest transcription factor Sox10 is preferentially expressed in triple-negative and metaplastic breast carcinomas. Hum Pathol 2013;44:959-65.

24. Artavanis-Tsakonas S, Rand MD, Lake RJ. Notch signaling: cell fate control and signal integration in development. Science 1999;284:770-6.

25. Bouras T, Pal B, Vaillant F, et al. Notch signaling regulates mammary stem cell function and luminal cell-fate commitment. Cell Stem Cell 2008;3:429-41. 
26. Zhu YT, Jia Y, Hu L, et al. Peroxisome-proliferatoractivated receptor-binding protein (PBP) is essential for the growth of active Notch4-immortalized mammary epithelial cells by activating SOX10 expression. Biochem J 2009;425:435-44.

27. Karamchandani JR, Nielsen TO, van de Rijn M, et al. Sox10 and S100 in the diagnosis of soft-tissue neoplasms. Appl Immunohistochem Mol Morphol 2012;20:445-50.

28. Ivanov SV, Panaccione A, Nonaka D, et al. Diagnostic SOX10 gene signatures in salivary adenoid cystic and breast basal-like carcinomas. Br J Cancer 2013;109:444-451.

29. Ohtomo R, Mori T, Shibata S, et al. SOX10 is a novel marker of acinus and intercalated duct differentiation in salivary gland tumors: a clue to the histogenesis for tumor diagnosis. Mod Pathol 2013;26:1041-50.

30. Miettinen M, McCue PA, Sarlomo-Rikala M, et al. Sox10-a marker for not only schwannian and melanocytic neoplasms but also myoepithelial cell tumors of soft tissue:

Cite this article as: Jin L, Qin C, Qi X, Hong T, Yang X, Zhu X. Clinicopathological significance of Sox10 expression in triplenegative breast carcinoma. Transl Cancer Res 2020;9(9):5603-5613. doi: $10.21037 /$ tcr-20-2634 a systematic analysis of 5134 tumors. Am J Surg Pathol 2015;39:826-35.

31. Tozbikian GH, Zynger DL. A combination of GATA3 and SOX10 is useful for the diagnosis of metastatic triplenegative breast cancer. Hum Pathol 2019;85:221-7.

32. He P, Jin X. SOX10 induces epithelial-mesenchymal transition and contributes to nasopharyngeal carcinoma progression. Biochem Cell Biol 2018;96:326-31.

33. Yin $\mathrm{H}$, Qin C, Zhao Y, et al. SOX10 is over-expressed in bladder cancer and contributes to the malignant bladder cancer cell behaviors. Clin Transl Oncol 2017;19:1035-44.

34. Kato M, Nishihara H, Hayashi H, et al. Clinicopathological evaluation of Sox 10 expression in diffuse-type gastric adenocarcinoma. Med Oncol 2017;34:8.

(English Language Editor: J. Jones) 